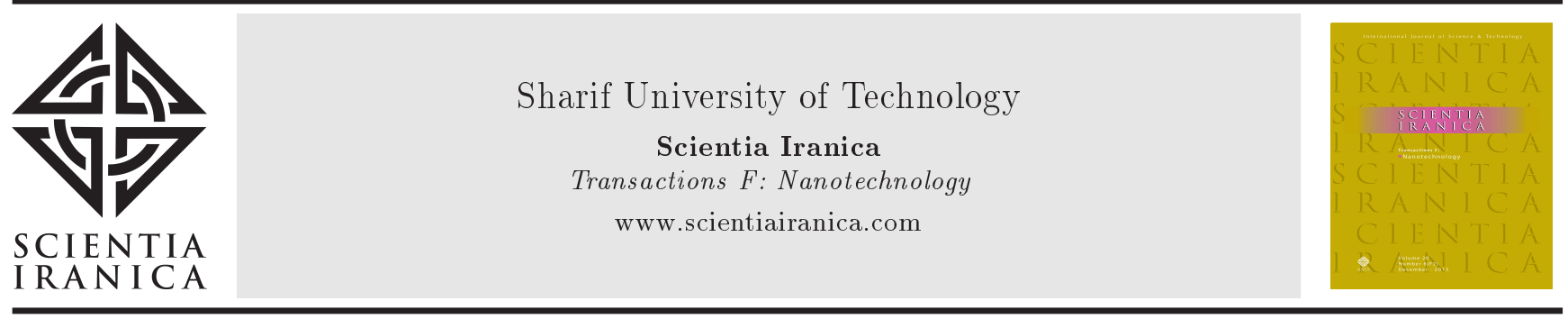

\title{
Impact of electrospun Tecophilic/gelatin scaffold biofunctionalization on proliferation of vascular smooth muscle cells
}

\author{
E. Vatankhah ${ }^{a, *}$, M.P. Prabhakaran ${ }^{b}$ and S. Ramakrishna ${ }^{b}$ \\ a. Department of Cellulose and Paper Technology, Faculty of New Technologies and Energy Engineering, Shahid Beheshti \\ University, Zirab Campus, Mazandaran, P.O. Box 4781173769, Iran. \\ b. Department of Mechanical Engineering, Faculty of Engineering, 2 Engineering Drive 3, National University of Singapore, \\ Singapore 117576, Singapore.
}

Received 8 August 2016; received in revised form 8 October 2016; accepted 19 December 2016

\section{KEYWORDS}

Electrospun scaffold;

Smooth muscle cells;

Proliferation;

Gelatin coating;

Fibronectin coating.

\begin{abstract}
Nanofibrous composite scaffolds based on Tecophilic (TP) and gelatin (gel) were electrospun, and further modification of their surfaces was performed by the adsorption of gelatin or fibronectin biomolecules. The ability of coated scaffolds to alter the proliferation rate of Smooth Muscle Cells (SMCs) was investigated via various assays and compared to cell proliferation on non-coated scaffolds. The results confirmed the potential of both coated and non-coated composite scaffolds to support SMC growth. Although the presence of fibronectin increased the proliferation, adsorbed gelatin could reduce the proliferation of SMCs. The success of a tissue-engineered vascular graft depends on the ability of the scaffold to control the proliferation rate of SMCs; thus, our study provides a better insight into the fabrication of functional constructs for vascular regeneration.
\end{abstract}

(C) 2017 Sharif University of Technology. All rights reserved.

\section{Introduction}

Extracellular matrix (ECM) is an intricate network of fibrillar and nonfibrillar macromolecules surrounding the cells consisting of collagen and elastin fibers along with proteoglycans and glycoproteins, providing both structural and regulatory functions to the cells. ECM provides mechanical support for the tissue and affects various aspects of cellular behavior including the adhesion, proliferation, and differentiation of cells through mechanical and biochemical signals $[1,2]$.

Electrospun nanofibrous scaffolds can mimic the physical dimension and architecture of native ECM in terms of geometry, morphology, and topography,

*. Corresponding author. Tel.: +981142451093 (44)

E-mail address: e_vatankhah@sbu.ac.ir (E. Vatankhah)

doi: $10.24200 /$ sci. 2017.4420 while the biological functionality of the nanofibers is considered a major challenge during the development of scaffolds for tissue engineering. Bioactive materials offer a convenient way to control the cell-material interactions [3] depending on the type of tissues or cells.

Vascular Smooth Muscle Cells (SMCs) regulate their phenotype in response to environmental chemical, physical, and mechanical signals. During our previous studies, we showed that an electrospun composite scaffold using TP and gelatin at a weight ratio of 70:30 could not only support the contractility of SMCs, but also provide appropriate mechanical properties comparable with those of native blood vessels [4,5]. TP is a family of hydrophilic polyether-based thermoplastic aliphatic polyurethane considered as thromboresistance and possesses inherent elasticity [6], suggesting its application as a vascular tissue-engineered graft. It was found that the addition of gelatin containing many integrin binding sites to TP could improve the potential 
of the composite scaffold to modulate the behavior of SMCs $[4,5]$.

The proliferation of SMCs rarely happens under normal physiological conditions; but, these cells can grow very fast under pathological conditions of some cardiovascular diseases such as atherosclerosis [7]. It is essential that a vascular tissue-engineered construct possess contractile SMCs, such that the proliferation of SMCs be controlled to prevent the recurrence of the disease [8]. Previously, we have found that TP(70)/gel(30) scaffold could direct SMCs towards a contractile phenotype due to its softness and presence of cell adhesion ligands within the fibers [4]. Here, we have studied the proliferation of SMCs in response to physical adsorption of biomolecules on the surface of $\mathrm{TP}(70) /$ gel(30) scaffolds. The differences in the interaction of SMCs cultured on TP(70)/gel(30) scaffolds coated with either gelatin or fibronectin in comparison with non-coated $\mathrm{TP}(70) / \operatorname{gel}(30)$ scaffolds are explored.

\section{Materials and methods}

\subsection{Materials}

TP was a kind gift from Lubrizol. Gelatin type A (300 Bloom) from porcine skin, gelatin solution from bovine skin, 1,1,1,3,3,3-hexafluoro-2-propanol (HFP), and Phosphate Buffered Saline (PBS) were all purchased from Sigma-Aldrich. Fibronectin solution was obtained from Invitrogen. Human aortic SMCs and Smooth Muscle Cell Medium (SMCM) were obtained from ScienCell Research Laboratories.

\subsection{Fabrication of nanofibrous scaffolds}

The method of electrospinning has been described in our previous studies $[4,5]$. Briefly, respective polymer solutions prepared in HFP, either TP alone $(8 \% \mathrm{w} / \mathrm{v})$ or $\mathrm{TP} /$ gel solution, $(8 \% \mathrm{w} / \mathrm{v}$, with the blend ratio of $70: 30 \mathrm{w} / \mathrm{w}, \mathrm{TP}(70) / \operatorname{gel}(30))$ were individually loaded into a $3 \mathrm{~mL}$ plastic syringe fitted with a $27 \mathrm{G}$ blunted stainless steel needle. A high voltage of $10 \mathrm{kV}$ was applied to the polymer solution fed at a constant flow rate of $1 \mathrm{~mL} / \mathrm{h}$ using a syringe pump. The distance between the syringe needle tip and a grounded flat collector wrapped in aluminum foil was kept at $12 \mathrm{~cm}$, and nanofibers were collected on the $15 \mathrm{~mm}$ cover slips placed on the collector. Electrospun scaffolds were transferred to a vacuum desiccator at ambient temperature for at least $48 \mathrm{~h}$ to eliminate residual solvents.

\subsection{Analysis of nanofibers}

The morphology of the electrospun scaffolds was observed under Scanning Electron Microscope (SEM; FEI-QUANTA 200FQ12, Netherlands) at an accelerating voltage of $10 \mathrm{kV}$. Before observation, each sample was coated with gold by a sputter coater (JEOL JFC-1600 Auto fine coater, Japan). Based on the
SEM images, fiber diameter was determined using image analysis program (Image J, National Institutes of Health, USA).

The surface roughness of the substrates was quantitatively evaluated using Atomic Force Microscopy (AFM, Dimension 3100, Digital Instruments, USA) in a dynamic force mode. Silicon nanoprobe tips (Nanosensors, Switzerland) with a resonant frequency near $330 \mathrm{kHz}$ were used. The Root Mean Square (RMS) roughness as one of the roughness parameters in three random areas of each surface with dimensions of $50 \mu \mathrm{m}$ $\times 50 \mu \mathrm{m}$ ( $x, y$ directions) analyzed at the scan rate of $0.5 \mathrm{~Hz}$ and 256 scanning lines was calculated and reported by Nanoscope imaging software (version 5.30, Digital Instrument, Inc.).

The chemical composition of the nanofibrous surface was characterized using ATR-FTIR spectroscopy (Thermo Nicolet, Waltham, MA). The transmittance of samples was recorded between $400 \mathrm{~cm}^{-1}$ and $4000 \mathrm{~cm}^{-1}$, with a resolution of $4 \mathrm{~cm}^{-1}$.

\subsection{Scaffold functionalization and cell seeding} Human aortic SMCs were normally cultured in a $150 \mathrm{~cm}^{2}$ cell-culture flask using Smooth Muscle Cell Medium (SMCM) containing basal medium complemented with $2 \%$ Fetal Bovine Serum (FBS), $1 \%$ Smooth Muscle Cell Growth Supplement (SMCGS), and $1 \%$ Penicillin/Streptomycin solution (P/S). The SMCs were incubated at $37^{\circ} \mathrm{C}$ in a humidified atmosphere containing $5 \% \mathrm{CO}_{2}$ for 7 days, and the culture medium was changed once in every 3 days.

The electrospun nanofibrous scaffolds collected on glass cover slips of $15-\mathrm{mm}$ diameter were placed in 24-well plates and pressed with a stainless steel ring to prevent swelling and shrinkage. The fibers were sterilized under UV light for $2 \mathrm{~h}$ and washed thrice with $\mathrm{PBS}$ for $5 \mathrm{~min}$ each in order to remove any residual solvent. The composite samples $(\mathrm{TP}(70) / \operatorname{gel}(30))$ were divided into three groups. Subsequently, electrospun $\mathrm{TP}$ and one group of $\mathrm{TP}(70) / \operatorname{gel}(30)$ scaffolds along with Tissue Culture Polystyrene (TCP) were immersed in $200 \mu \mathrm{L}$ of SMCM overnight before cell seeding. Furthermore, two other groups of composite scaffolds were submerged in $200 \mu \mathrm{L}$ of either gelatin solution or fibronectin solution (diluted in SMCM to obtain $10 \mu \mathrm{g} /$ well) overnight before cell seeding. The cultured SMCs were trypsinized by trypsin-EDTA, counted with trypan blue using hemocytometer, and $200 \mu \mathrm{L}$ of cell suspension containing $1 \times 10^{4}$ cells was pipetted onto the upper surface of each well and allowed to be attached for $24 \mathrm{~h}$ before adding extra SMCM to reach $1 \mathrm{~mL}$ medium/well.

\subsection{Cell viability}

After 7 days of culturing cells on the scaffolds or control wells, cells were stained using the Live/Dead 
Viability/Cytotoxicity Kit for mammalian cells (Molecular Probes, Invitrogen) containing calcein AM and ethidium homodimer-1 (EthD-1) to stain live and dead cells with green and red fluorescent dyes, respectively. The staining solution was prepared by adding $20 \mu \mathrm{L}$ of $2 \mathrm{mM}$ EthD-1 solution and $5 \mu \mathrm{L}$ of $4 \mathrm{mM}$ calcein to $10 \mathrm{~mL}$ of PBS. Cell-scaffold constructs cultured for 7 days were washed with PBS and incubated with $200 \mu \mathrm{L}$ of staining solution for $30 \mathrm{~min}$ at $37^{\circ} \mathrm{C}$. Then, samples were again washed with $\mathrm{PBS}$ and imaged with a Leica DM IRB fluorescent microscope.

\subsection{Cell proliferation}

The adhesion and proliferation of cultured SMCs on coated and non-coated scaffolds as well as TCP were evaluated by the colorimetric MTS (3-(4, 5dimethylthiazol-2-yl)-5-(3-carboxymethoxyphenyl)-2(4-sulfophenyl)-2H- tetrazolium) assay using CellTiter 96 AQueous One solution (Promega, Madison, WI). After culturing the cells for a period of 1, 4, 7, and 10 days, cell-seeded constructs were rinsed with PBS to remove unattached cells and also were incubated with $1 \mathrm{~mL}$ of serum-free medium containing $20 \%$ of MTS reagent at $37^{\circ} \mathrm{C}$ for $3 \mathrm{~h}$. Absorbance of the obtained dye was measured using a spectrophotometric plate reader (FLUOstar Optima, BMG Lab Technologies, Offenburg, Germany) at $490 \mathrm{~nm}$.

\subsection{Cell morphology}

After 7 days of cell culture, morphological characteristics of SMCs seeded on electrospun scaffolds and TCP were studied by SEM. Cell-seeded constructs were washed with PBS and fixed with $3 \%$ glutaraldehyde (Sigma-Aldrich) for $3 \mathrm{~h}$. Fixed samples were further rinsed with deionized water and exposed to a gradient of ethanol (50\%, 70\%, 90\%, and 100\%) followed by treating with hexamethyldisilazane. Finally, specimens were air dried in a fume hood and examined using SEM.

\subsection{Statistical analysis}

All data presented were expressed as mean \pm Standard Deviation (SD) of the mean. One-way ANOVA combined with Turkey post hoc tests was carried out for multiple comparisons of different samples. A value of $p<0.05$ was considered statistically significant.

\section{Results and discussion}

\subsection{Characterization of nanofibrous scaffolds}

Using electrospinning technique, nanoscale and beadless TP fibers and TP/gel blended fibers were fabricated. Figure 1 provides SEM micrographs of porous
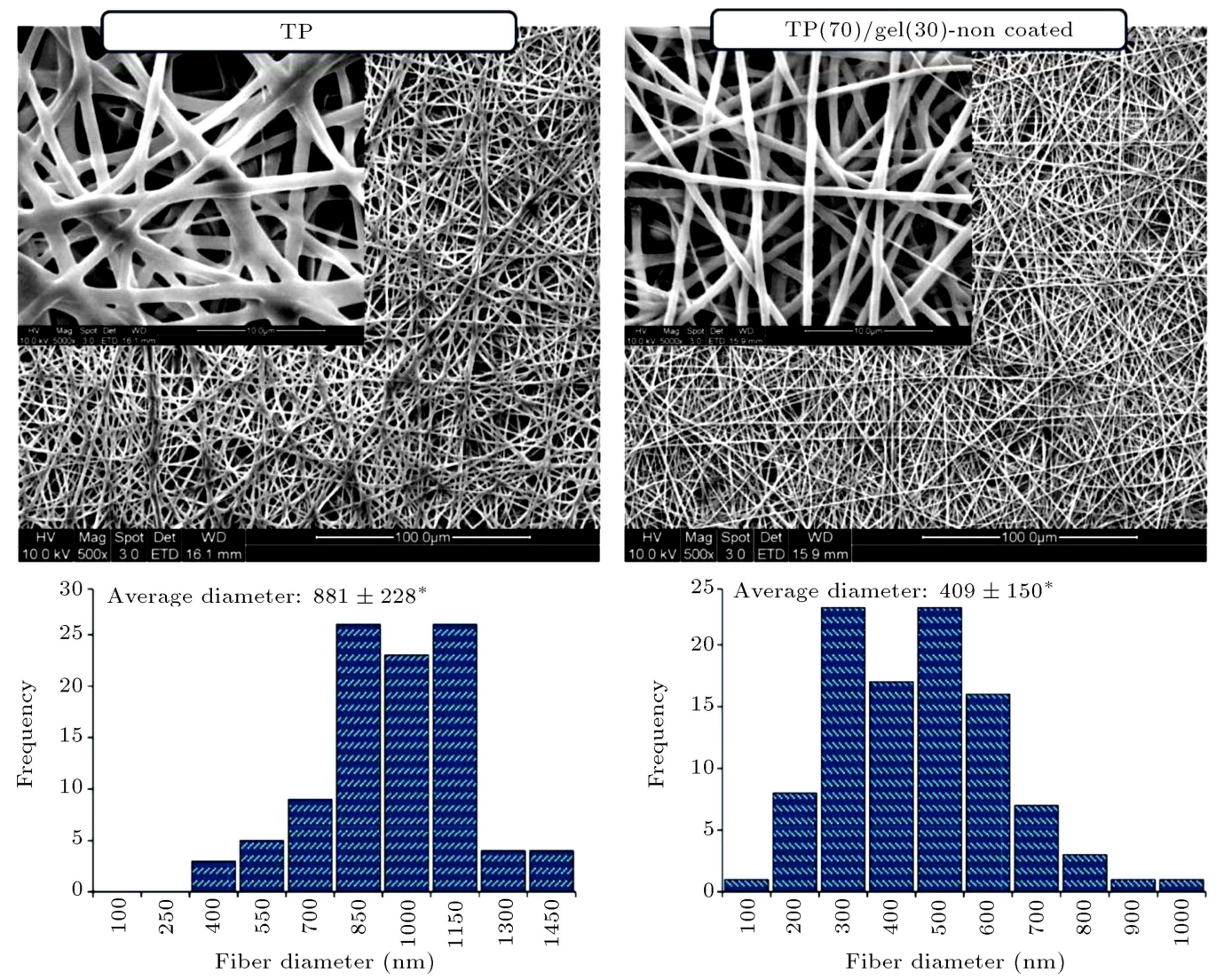

Figure 1. Morphology and fiber diameter distribution histogram of electrospun TP and TP(70)/gel(30) scaffolds.

* Significant differences at $p<0.05$. 


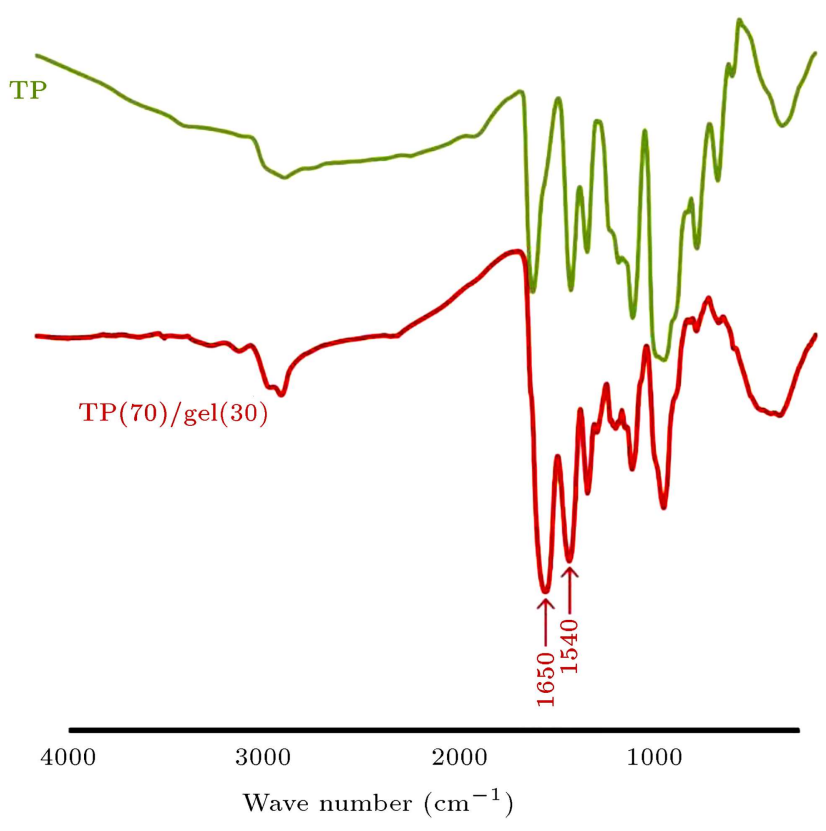

Figure 2. FTIR spectra of electrospun TP and $\mathrm{TP}(70) / \mathrm{gel}(30)$ nanofibers.

and uniform interconnected structures of electrospun scaffolds along with fiber diameter and size distribution.

SEM images clearly demonstrated that the fiber diameter was significantly decreased from $881 \pm 228 \mathrm{~nm}$ to $409 \pm 150 \mathrm{~nm}$, and the diameter distribution was broadened after adding gelatin to composition, which might be due to the role of amino acids provided by gelatin to improve the stretching force and selfrepulsion and decrease the viscosity of the composite solution [9].

The arterial wall tissue possesses high degree of nanometer surface roughness [10], and electrospun fibers can provide surface roughness on a nanometer scale [11]. The roughness of a biomaterial-based graft is an important factor due to its effect on various cellular behaviors such as protein adsorption, cell attachment, and cell proliferation $[12,13]$. Similar to the results reported by Milleret et al. [14], surface roughness increased with increasing constituent fiber diameter (Figure 2). The average RMS roughnesses of the electrospun TP and TP(70)/gel(30) surfaces were $861 \pm 90 \mathrm{~nm}$ and $514 \pm 37 \mathrm{~nm}$, respectively, which corroborated a significantly smoother surface of the composite scaffold compared to pure one.

The FTIR spectra of the electrospun scaffolds are shown in Figure 3. For pure TP, the bands at about $2935 \mathrm{~cm}^{-1}$ and $2856 \mathrm{~cm}^{-1}$ were attributed to asymmetric and symmetric stretching vibrations of $\mathrm{CH}_{2}$-, respectively. Urethane $\mathrm{C}=\mathrm{O}$ stretching was visible at $1703 \mathrm{~cm}^{-1}$ and the bands at $1530 \mathrm{~cm}^{-1}$ and $1310 \mathrm{~cm}^{-1}$ were both responsible for urethane $\mathrm{N}-\mathrm{H}$ bending and $\mathrm{C}-\mathrm{N}$ stretching. In addition, the band at
$1450 \mathrm{~cm}^{-1}$ was assigned to $-\mathrm{CH}_{2}$ - bending vibration. The strong characteristic bands appearing at about $1110 \mathrm{~cm}^{-1}$ and $1080 \mathrm{~cm}^{-1}$ arose from stretching of $\mathrm{C}-$ $\mathrm{O}-\mathrm{C}$ bond, respectively, in aliphatic ether of soft and hard segments of TP. The composite scaffold exhibited similar IR peaks with those of the pure TP matrix [1517]. In addition, two typical peaks were observed at $1650 \mathrm{~cm}^{-1}$ and $1540 \mathrm{~cm}^{-1}$ of the composite scaffold spectrum corresponding to amide I band and amide II band of the gelatin, respectively $[9,18]$.

\subsection{Cell growth and morphology}

Using biomolecules mimicking the natural ECM is a promising approach to tissue engineering. The behavior of cells, including cell adhesion, proliferation, and differentiation in contact with bioactive motifs of ECM, or other biomolecules attached to the surface of the scaffold, can partially influence the profile of tissue reconstruction. Vasoactivity of contractile SMCs through vascular regeneration may enable the production of functional tissue-engineered blood vessels. We explained the potential of the scaffolds to control the phenotypic modulation of SMCs in our previous studies and indicated the preservation of SMCs contractility on electrospun $\mathrm{TP}(70) /$ gel(30) scaffolds $[4,5]$. While the proliferation of SMCs seeded on scaffolds is generally essential to regenerate a vascular tissue, uncontrollable proliferation in the implanted graft can cause the thickening of the vessel wall and narrowing of the vessel lumen [7]. In this study, we have focused on the surface modification of electrospun $\mathrm{TP}(70) / \operatorname{gel}(30)$ scaffolds with the physical adsorption of either fibronectin or gelatin onto the surface to investigate their effects on SMCs proliferation.

The survival and retention of SMCs on the coated and non-coated composite scaffolds were determined by cell viability assay after 7 days of cell culture. In this assay, live cells are distinguished by the presence of ubiquitous intracellular esterase activity determined by the enzymatic conversion of the virtually nonfluorescent cell-permeant calcein AM to the intensely fluorescent calcein. The polyanionic dye calcein is well retained within the live cells, producing an intense uniform green fluorescence in live cells. EthD-1 enters cells with damaged membranes and undergoes a 40fold enhancement of fluorescence, thereby producing a bright red fluorescence in dead cells upon binding to nucleic acids. EthD-1 is excluded by the intact plasma membrane of live cells. As evident in Figure 4, cells survived well and a small number of dead cells were observed on all scaffolds confirming their biocompatibility without producing toxic effects; however, the lack of sufficient cell recognition ligands on the pure TP scaffold resulted in the poor attachment and proliferation of cells on this construct. SMCs demonstrated aligned orientation and parallel alignment on composite scaf- 


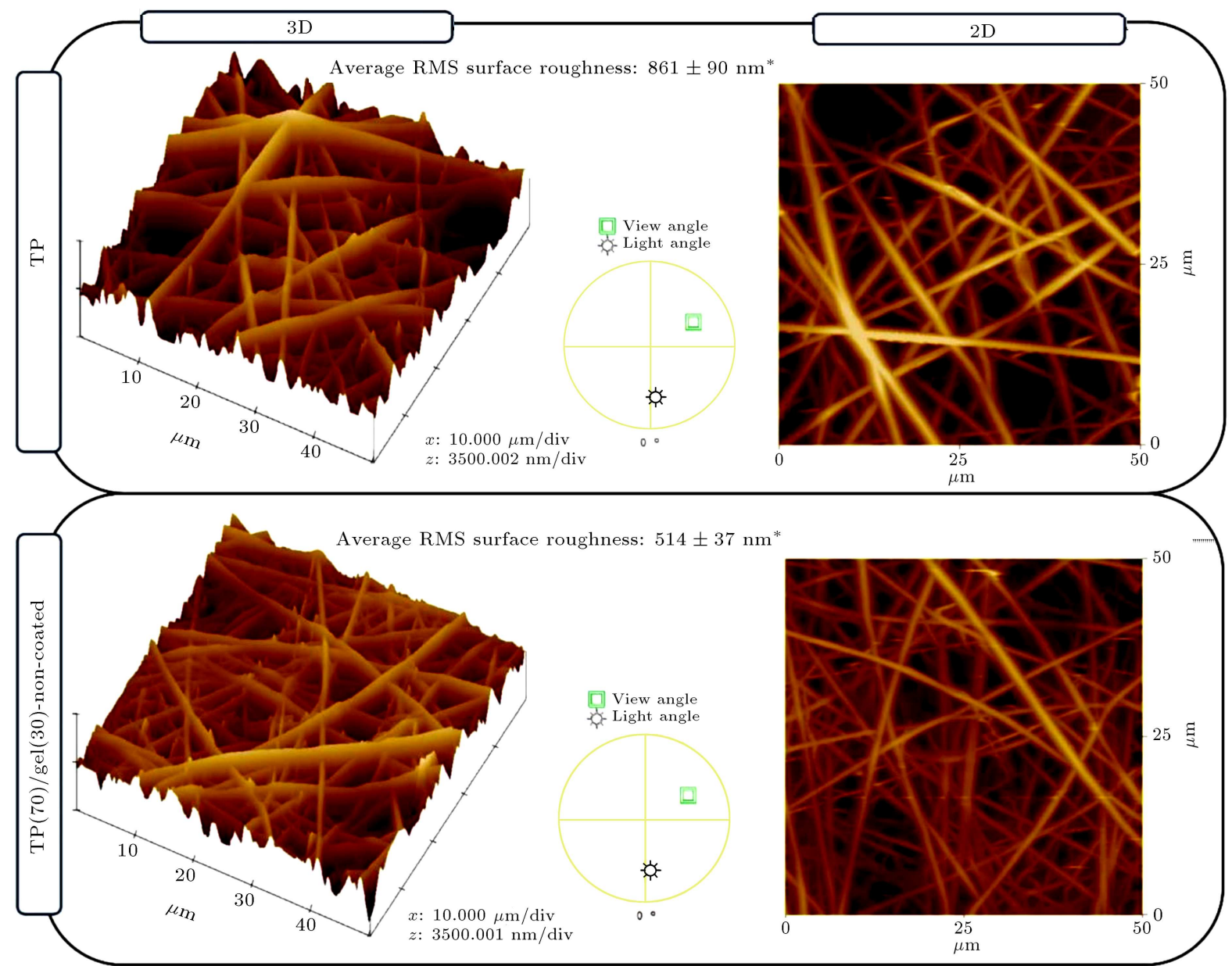

Figure 3. Representative of $3 \mathrm{D}$ and $2 \mathrm{D}$ AFM micrographs of electrospun TP and TP(70)/gel(30) nanofibers. * Significant differences at $p<0.05$.
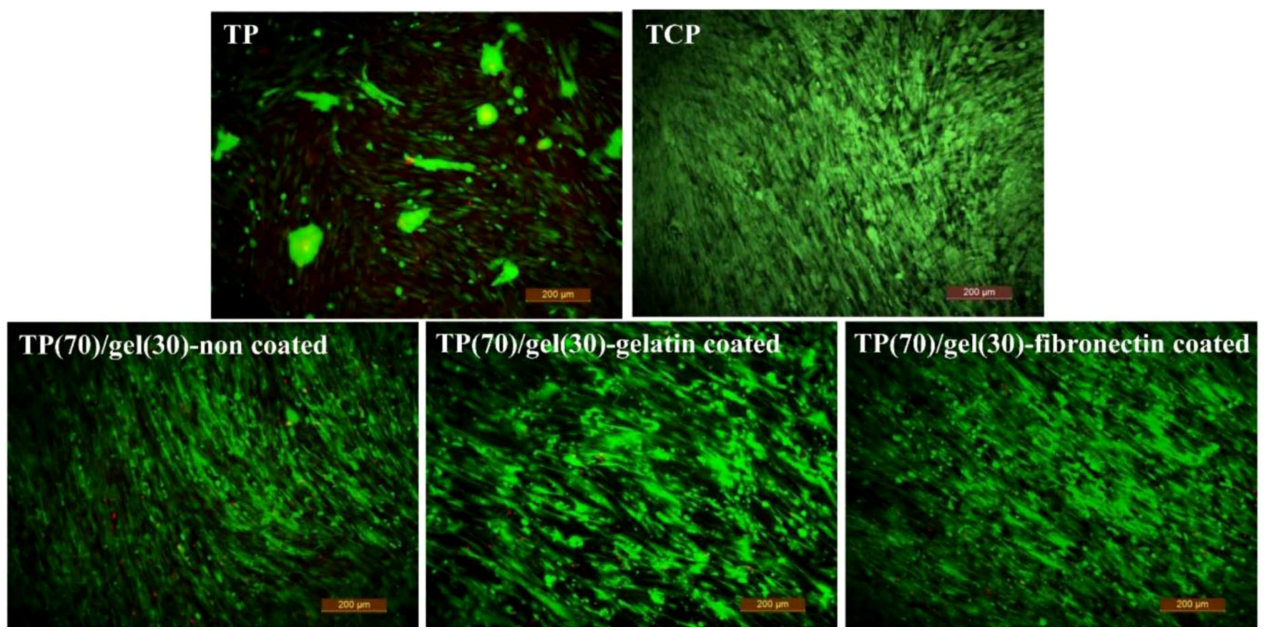

Figure 4. Cell survival and morphology of SMCs grown on electrospun TP, TP(70)/gel(30)-non-coated, $\mathrm{TP}(70) /$ gel(30)-gelatin coated, TP(70)/gel(30)-fibronectin coated scaffolds and TCP.

folds rather than on pure TP and TCP, confirming the maintenance of contractile phenotype of SMCs on coated and non-coated electrospun TP(70)/gel(30) scaffolds. The growth and mitochondrial metabolic activity of SMCs on the scaffolds assessed by MTS assay after 1, 4, 7, and 10 days of cell culture are shown in Figure 5. It was observed that cells on all scaffolds adhered to some extent, began to proliferate after culturing for $1 \mathrm{day}$, and continued to increase in number over time. The results illustrated that 


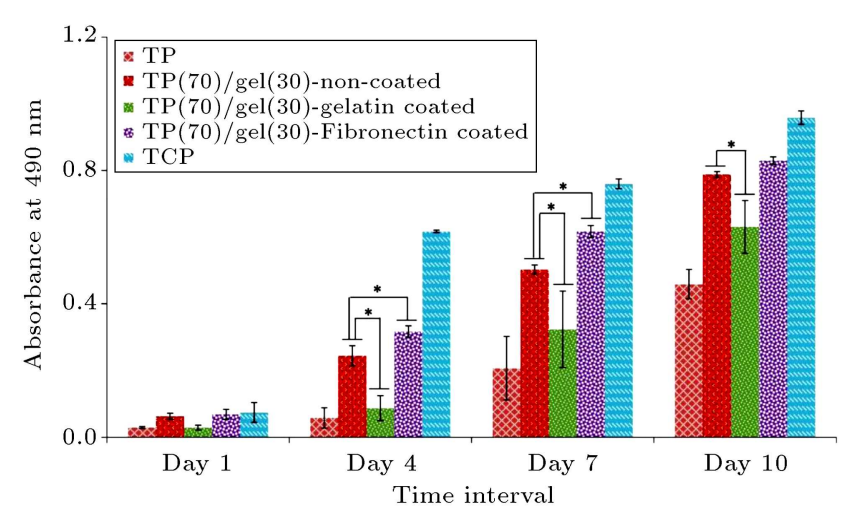

Figure 5. Proliferation of SMCs on electrospun TP, $\mathrm{TP}(70) /$ gel(30)-non-coated, TP(70)/gel(30)-gelatin coated, $\operatorname{TP}(70) /$ gel(30)-fibronectin coated nanofibers and TCP. * Significant differences against cell proliferation on $\mathrm{TP}(70) /$ gel(30)-non-coated scaffolds at $p<0.05$.

non-coated, gelatin coated, and fibronectin coated composite scaffolds were able to support SMCs proliferation in a greater level than on pure TP scaffolds. The proliferation of SMCs on TP(70)/gel(30)gelatin coated and $\mathrm{TP}(70) /$ gel(30)-fibronectin coated scaffolds displayed significantly lower and higher levels, respectively, compared to $\mathrm{TP}(70) / \operatorname{gel}(30)$-noncoated scaffold on days 4 and 7 . After culturing for 10 days, significant differences in cell number on noncoated and gelatin-coated scaffolds were also observed. Although there was an increase in cell proliferation on fibronectin coated scaffolds compared to non-coated ones, this increase was not statistically meaningful on day 10. Generally, among all the composite scaffolds, the highest cell proliferation rate was obtained on fibronectin coated constructs, while it was significantly lower than proliferation on TCP at days 4, 7, and 10 . These findings suggest that $\mathrm{TP}(70) / \operatorname{gel}(30)$ scaffolds can provide a suitable substrate for SMCs, and physical adsorption of various biomolecules on their surface enables researchers to obtain a desirable proliferation rate. This finding, in accordance with those of other researchers [19-21], confirmed the behavioral regulation of the cells cultured on scaffolds via the involvement of specific integrin/ECM interaction. Additionally, as suggested by Kim et al., it was observed that the initial interaction of SMCs with the scaffold can influence the long-term behavior of the cells due to setting off a chain of signaling events through the initial cell contact with the scaffold led to long-term changes in gene expression and tissue composition [21].

The morphology of SMCs on electrospun scaffolds was further investigated via SEM. Results presented in Figure 6 revealed SMCs attachment to composite
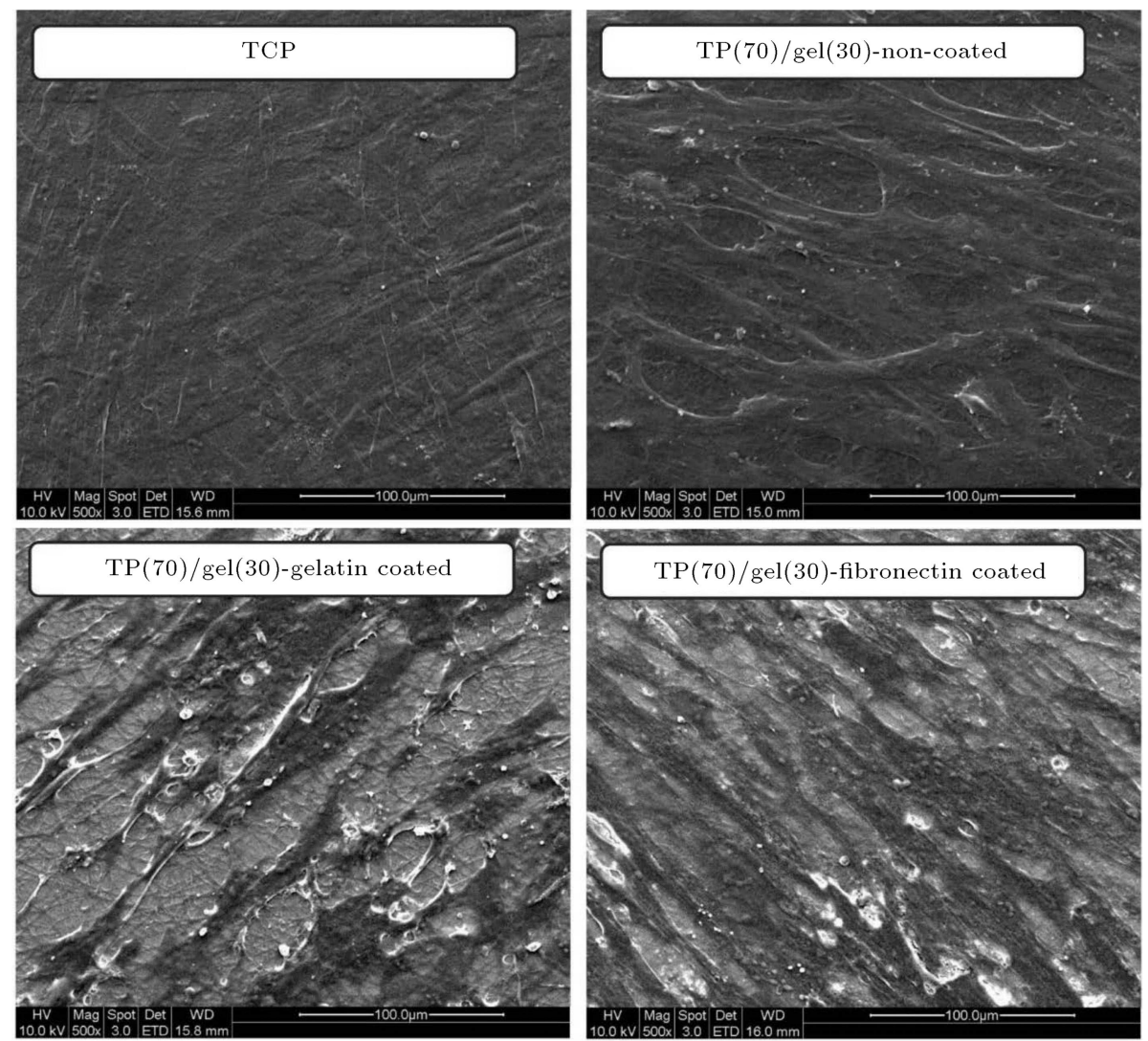

Figure 6. Morphology of SMCs on electrospun TP, TP(70)/gel(30)-non-coated, TP(70)/gel(30)-gelatin coated, $\mathrm{TP}(70) / g e l(30)$-fibronectin coated scaffolds and TCP. 
scaffolds after 7 days of cell culture, consistent with results of viability and MTS assays. Although the SMCs spread on coated and non-coated constructs showed similar morphologies with aligned organization compared to the cell morphology appearing on TP and $\mathrm{TCP}$, the cell proliferation rate on these composite constructs (covered area by cells) was their main difference.

The proliferation of $\mathrm{CMCs}$ on composite scaffolds increased in the following order; $\operatorname{TP}(70) / \operatorname{gel}(30)$ fibronectin coated $>\mathrm{TP}(70) /$ gel(30)-noncoated $>\mathrm{TP}$ (70)/gel(30)- gelatin coated, all suggest the ability of biomolecules to control proliferation of SMCs seeded on the same scaffolds with only different biomolecules modifying their surfaces. This simple method might have a valuable outcome for the appropriate regeneration of vascular tissue-engineered grafts.

\section{Conclusion}

This study demonstrated the important role of the specific cell adhesion biomolecules, including gelatin and fibronectin, coupled with the surface of electrospun $\mathrm{TP}(70) /$ gel(30) scaffolds in the control of SMC proliferation to engineer new functional vascular tissues. Results showed that fouling of surface by adsorption of fibronectin biomolecules might promote the surface to enhance cell growth, while the presence of gelatin biomolecules on the surface of the scaffold leads to a decreasing propensity in the proliferation of SMCs. Therefore, $\operatorname{TP}(70) /$ gel(30)-gelatin coated scaffolds can be a more attractive candidate for the development of a functional vascular graft with low proliferated SMCs in the wall.

\section{References}

1. Dekkers, B.G.J., Schaafsma, D., Nelemans, S.A., Zaagsma, J. and Meurs, H. "Extracellular matrix proteins differentially regulate airway smooth muscle phenotype and function", Am. J. Physiol. Lung Cell. Mol. Physiol., 292(6), pp. L1405-L1413 (2007).

2. Tresoldi, I., Oliva, F., Benvenuto, M., Fantini, M., Masuelli, L., Bei, R. and Modesti, A. "Tendon's ultrastructure", Muscles Ligaments Tendons J., 3(1), pp. 2-6 (2013).

3. Mann, B.K., Tsai, A.T., Scott-Burden, T. and West, J.L. "Modification of surfaces with cell adhesion peptides alters extracellular matrix deposition", Biomaterials, 20(23-24) pp. 2281-2286, (1999).

4. Vatankhah, E., Prabhakaran, M.P., Semnani, D., Razavi, S., Zamani, M. and Ramakrishna, S. "Phenotypic modulation of smooth muscle cells by chemical and mechanical cues of electrospun tecophilic/gelatin nanofibers", ACS Appl. Mater. Interfaces, 6(6), pp. 4089-4101 (2014).
5. Vatankhah, E., Prabhakaran, M.P., Semnani, D., Razavi, S., Morshed, M. and Ramakrishna, S. "Electrospun tecophilic/gelatin nanofibers with potential for small diameter blood vessel tissue engineering", Biopolymers, 101(12), pp. 1165-1180 (2014).

6. Melaiye, A., Sun, Z., Hindi, K., Milsted, A., Ely, D., Reneker, D.H., Tessier, C.A. and Youngs, W.J. "Silver(I)-imidazole cyclophane gem-diol complexes encapsulated by electrospun tecophilic nanofibers: formation of nanosilver particles and antimicrobial activity", J. Am. Chem. Soc., 127(7), pp. 2285-2291 (2005).

7. Chan-Park, M.B., Shen, J.Y., Cao, Y., Xiong, Y., Liu, Y., Rayatpisheh, S., Kang, G.C.-W. and Greisler, H.P. "Biomimetic control of vascular smooth muscle cell morphology and phenotype for functional tissueengineered small-diameter blood vessels", J. Biomed. Mater. Res. A., 88A(4), pp. 1104-1121 (2009).

8. Stegemann, J.P., Hong, H. and Nerem, R.M. "Mechanical, biochemical and extracellular matrix effects on vascular smooth muscle cell phenotype", J. Appl. Physiol., 98(6), pp. 2321-2327 (2005).

9. Meng, Z.X., Wang, Y.S., Ma, C., Zheng, W., Li, L. and Zheng, Y.F. "Electrospinning of PLGA/gelatin randomly-oriented and aligned nanofibers as potential scaffold in tissue engineering", Mater. Sci. Eng., C, 30(8), pp. 1204-1210 (2010).

10. Miller, D.C., Thapa, A., Haberstroh, K.M. and Webster, T.J. "Endothelial and vascular smooth muscle cell function on poly(lactic-co-glycolic acid) with nanostructured surface features", Biomaterials, 25(1), pp. 53-61 (2004).

11. Zamani, F., Amani-Tehran, M., Latifi, M. and Shokrgozar, M.A. "The influence of surface nanoroughness of electrospun PLGA nanofibrous scaffold on nerve cell adhesion and proliferation", J. Mater. Sci. Mater. Med., 24(6), pp. 1551-1560 (2013).

12. Anselme, K., Bigerelle, M., Noel, B., Dufresne, E., Judas, D., Iost, A. and Hardouin, P. "Qualitative and quantitative study of human osteoblast adhesion on materials with various surface roughnesses", $J$. Biomed. Mater. Res., 49(2), pp. 155-166 (2000).

13. Jaiswal, A.K., Kadam, S.S., Soni, V.P. and Bellare, J.R. "Improved functionalization of electrospun PLLA/gelatin scaffold by alternate soaking method for bone tissue engineering", Appl. Surf. Sci., 268, pp. 477-488 (2013).

14. Milleret, V., Hefti, T., Hall, H., Vogel, V. and Eberli, D. "Influence of the fiber diameter and surface roughness of electrospun vascular grafts on blood activation", Acta Biomater., 8(12), pp. 4349-4356 (2012).

15. Srichatrapimuk, V.W. and Cooper, S.L. "Infrared thermal analysis of polyurethane block polymers", $J$. Macromol. Sci., Phys., 15(2), pp. 267-311 (1978). 
16. McCarthy, S.J., Meijs, G.F., Mitchell, N., Gunatillake, P.A., Heath, G., Brandwood, A. and Schindhelm, K. "In-vivo degradation of polyurethanes: transmissionFTIR microscopic characterization of polyurethanes sectioned by cryomicrotomy", Biomaterials, 18(21), pp. 1387-1409 (1997).

17. Sung Eun, K., Dong Nyoung, H., Jung Bok, L., Jong Ryul, K., Sang Hyuk, P., Seong Ho, J. and Il Keun, K. "Electrospun gelatin/polyurethane blended nanofibers for wound healing", Biomed. Mater., 4(4), p. 044106 (2009).

18. Ki, C.S., Baek, D.H., Gang, K.D., Lee, K.H., Um, I.C. and Park, Y.H. "Characterization of gelatin nanofiber prepared from gelatin-formic acid solution", Polymer, 46(14), pp. 5094-5102 (2005).

19. Hedin, U., Bottger, B.A., Forsberg, E., Johansson, S. and Thyberg, J. "Diverse effects of fibronectin and laminin on phenotypic properties of cultured arterial smooth muscle cells", J. Cell Biol., 107(1), pp. 307319 (1988).

20. Thyberg, J. and Hultgårdh-Nilsson, A. "Fibronectin and the basement membrane components laminin and collagen type IV influence the phenotypic properties of subcultured rat aortic smooth muscle cells differently", Cell Tissue Res., 276(2), pp. 263-271 (1994).

21. Kim, B.-S., Nikolovski, J., Bonadio, J., Smiley, E. and Mooney, D.J. "Engineered smooth muscle tissues: regulating cell phenotype with the scaffold", Exp. Cell Res., 251(2), pp. 318-328 (1999).

\section{Biographies}

Elham Vatankhah joined the Department of New Technologies Engineering at Shahid Beheshti University as an Assistant Professor in 2016. She completed her PhD in Textile Engineering at Isfahan University of Technology. Her research interests include biomedical textiles and nanofibrous biomaterials for tissue engineering and drug delivery.

Molamma P. Prabhakaran received her $\mathrm{PhD}$ in Chemistry from the Faculty of Science in The National University of Singapore (NUS) and worked as a Research Fellow with the Faculty of Engineering in NUS. Her research interests include fabrication of biomimetic scaffolds, modifications together with application of nanofibers for tissue engineering of the skin, bone, nerve, and cardiac.

Seeram Ramakrishna, FREng, FBSE, is the Director of Center for Nanofibers \& Nanotechnology at the National University of Singapore (NUS). He is a highly cited Researcher in Materials Science (www.highlycited.com). He is among the World's Most Influential Scientific Minds (Thomson Reuters). He coauthored 1,000 articles with 60,000 citations and 115 H-index. His research outcomes have been translated into products. He is a Fellow of UK Royal Academy of Engineering (FREng); Biomaterials Science and Engineering (FBSE); American Association of the Advancement of Science (AAAS), and American Institute for Medical \& Biological Engineering (AIMBE). He is a recipient of IFEES President award-Global Visionary; Chandra P Sharma Biomaterials Award; Nehru Fellowship; LKY Fellowship; NUS Outstanding Researcher Award; IES Outstanding Engineer Award; and ASEAN Outstanding Engineer Award. He received $\mathrm{PhD}$ degree from the University of Cambridge, UK, and The General Management Training from Harvard University, USA. He is a member of ISO/TC national committee on Medical Devices, Singapore. He chairs the Future of Manufacturing Technical Committee of Institution of Engineers Singapore. He is an Editor of Current Opinion in Biomedical Engineering. 